\title{
Prediction of post partum thyroid dysfunction: can it be improved?
}

\author{
Johannes L Kuijpens, Victor J Pop ${ }^{1}$, Huib L Vader ${ }^{2}$, Hemmo A Drexhage ${ }^{3}$ and Wilmar M Wiersinga ${ }^{4}$ \\ Municipal Health Service Southeast Brabant, Valkenswaard, The Netherlands, ${ }^{1}$ Department of Social and Behavioral Sciences, University of Tilburg, \\ The Netherlands, ${ }^{2}$ Clinical Laboratories, St Joseph Hospital, Veldhoven, The Netherlands, ${ }^{3}$ Department of Immunology, Erasmus University, Rotterdam, \\ The Netherlands and ${ }^{4}$ Department of Endocrinology AMC, University of Amsterdam, The Netherlands
}

(Correspondence should be addressed to J L Kuijpens, Department of Public Health, Municipal Health Service Southeast Brabant, PO Box 135, 5550 AC Valkenswaard, The Netherlands)

\begin{abstract}
Background: Screening pregnant women for thyroid peroxidase antibodies (TPOAb) to identify those at risk for post partum thyroid dysfunction (PPTD) is controversial, mainly because of the low positive predictive value (ppv) of TPOAb.

Objectives: To evaluate if the ppv of TPOAb can be enhanced, either by taking into account the time of TPOAb testing, or by combining this parameter with other putative determinants of PPTD such as smoking, family history or other autoimmune diseases.

Methods: A prospective study was performed in the Kempenland region (southeastern Netherlands). Three hundred and ten unselected women were visited at 12 and 32 weeks gestation and 4, 12, 20, 28 and 36 weeks post partum. Serial thyroid stimulating hormone (TSH), free thyroxine $\left(\mathrm{fT}_{4}\right)$ and TPOAb testing was performed. Thyroid dysfunction (TD) was defined as abnormal TSH either in combination

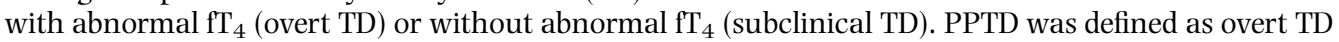
post partum. Multivariate regression analysis was performed for determining independent risk factors for PPTD. The sensitivity and specificity of TPOAb at different time points and at different concentrations were calculated and presented in receiver operating characteristic (ROC) curves. Women who had experienced PPTD were followed for 2.5-3 years.

Results: Data from 291 women were available for analysis. Serum $\mathrm{fT}_{4}$ declined during pregnancy and returned to baseline values post partum. TD in gestation was present in 23 women (7.9\%): serum TSH was transiently decreased in 13 (6 had overt gestational thyrotoxicosis $(2.1 \%)$ ) and increased in $10(2$ had TPOAb). Both point prevalence and concentration of TPOAb decreased during gestation and returned to baseline levels within 12 weeks post partum. TD in post partum was present in 36 women (12.4\%): 21 had subclinical and 15 overt TD. Out of the 15 women with overt TD (incidence of PPTD: $5.2 \%) 10$ were positive for TPOAb (TPOAb+); 9 had thyrotoxicosis $(4 \mathrm{TPOAb}+$ ), 5 hypothyroidism ( 5 $\mathrm{TPOAb}+$ ) and 1 thyrotoxicosis followed by hypothyroidism $(\mathrm{TPOA} b+)$. Independent risk factors for PPTD were TPOAb (relative risk $(\mathrm{RR})=27.2)$, bottle feeding $(\mathrm{RR}=11.1)$ and smoking habits (ever smoked: $\mathrm{RR}=3.1$; women with PPTD had smoked more cigarettes for a longer period of time). The sensitivity of TPOAb testing was highest at 12 weeks gestation (0.67). The ppv of TPOAb was $0.31-0.75$ (depending on time of testing and concentration), increasing slightly to $0.38-0.80$ when combined with bottle feeding or smoking habits. There appeared to be an autoimmune form of PPTD in 2/3 of cases and a nonautoimmune form; women with the autoimmune form were at risk for developing permanent hypothyroidism.

Conclusions: A maximum of 2/3 of PPTD cases can be predicted from the presence of TPOAb because 1/3 remained negative for TPOAb. The most appropriate time for TPOAb testing is in the first trimester of pregnancy. The combination of TPOAb testing with anamnestic determinants of PPTD does not increase ppv substantially.
\end{abstract}

European Journal of Endocrinology 139 36-43

\section{Introduction}

Post partum thyroid dysfunction (PPTD) was recognized as a clinical entity in the 1970s $(1,2)$. The incidence of PPTD in the general population is $4-8 \%(3-6)$. PPTD is thought to be part of the spectrum of autoimmune thyroid diseases (AITD) and is caused, in the majority of cases, by a lymphocytic thyroiditis exacerbated in the first year post partum as the result of a 'rebound' from the suppressed immune system during pregnancy (4, 6). Fifty percent of pregnant women positive for thyroid peroxidase antibodies (TPOAb, identical to microsomal 
antibodies (MsAb)), will develop PPTD (3-6). PPTD is clinically characterized by symptoms associated with hyper- and/or hypothyroidism and depressive symptoms $(4-8)$.

TPOAb are strongly related with PPTD: women positive for TPOAb have a relative risk (RR) of 20-80 for developing PPTD $(3,5)$. However, the positive predictive value of TPOAb is about $40-60 \%$, and one out of four women with PPTD does not have detectable TPOAb during pregnancy or in the post partum period $(3,5)$. Whether screening all pregnant women for TPOAb in order to predict PPTD is worthwhile remains controversial. Some promote screening because of the risk of abortion, the risk of developing hypothyroidism in gestation or in future life, or the risk of PPTD (9-11). Others state that because of the low predictive value of TPOAb and the absence of severe clinical symptoms in the majority of women with PPTD, screening is not justified $(12,13)$. Screening, however, requires in the first place a test with high sensitivity to identify persons at risk, a high specificity to prevent false positive cases (which might be a great problem in diseases with a low prevalence) and a high predictive value.

The objectives of the present study, therefore, were to evaluate if the predictive value of TPOAb for PPTD can be enhanced by taking into account the time of TPOAb testing together with other putative determinants of PPTD (such as family history, previous autoimmune diseases, smoking behavior).

\section{Subjects and methods}

\section{Subjects}

The study was performed between January 1994 and April 1996 in the Kempenland region in the southeastern part of the province of Noord-Brabant, The Netherlands. The iodine intake is low-normal in this region: the mean daily urinary iodide excretion is $111 \pm 70 \mu \mathrm{g}(14)$.

Four hundred and forty eight consecutive women not using thyroid drugs at their first visit to the local midwife or obstetrical department of the St Joseph Hospital, Veldhoven (covering $90 \%$ of all pregnancies in the region), were asked to participate. Three hundred and ten women $(69 \%)$ consented. Women who experienced spontaneous miscarriages or stillbirth were asked to continue the study; women pregnant again within 6 months after delivery were excluded from analysis. All women were visited at home at 12 and 32 weeks gestation and at 4,12, 20, 28 and 36 weeks post partum. A personal and family history for thyroid and autoimmune disease was performed; information on medication, smoking habits and alcohol intake was obtained. In the first post partum visit the obstetrical history was performed. Signs or symptoms of thyrotoxicosis or hypothyroidism were looked for at every visit. Venous blood samples were collected into Vacutainer tubes $(8 \mathrm{ml})$ for thyroid function and thyroid antibody testing every visit; serum was stored at $-20^{\circ} \mathrm{C}$.

Permission for the study was obtained from the Medical Ethics Committee of the Academic Medical Centre, University of Amsterdam.

\section{Methods}

Thyroid function tests The concentration of thyroid stimulating hormone (TSH) was measured by an immunometric technique, based on enhanced luminescence (Kodak Amerlite TSH-30, Kodak Clinical Diagnosticts Ltd, Amersham, Bucks, UK). The reference interval for TSH was $0.15-2.0 \mathrm{mU} / \mathrm{l}$, as defined for 225 non-pregnant women in the age group of 20-40 years and living in the same region, using the IFCC recommendations for defining reference values (15). The interassay coefficients of variation were 20, 4.8, 6.3 and $5.1 \%$ at concentrations of $0.04,0.68,8.2$ and $29.2 \mathrm{mU} / \mathrm{l}$ respectively.

Free thyroxine $\left(\mathrm{fT}_{4}\right)$ was determined by using the Kodak Amerlite MAB $\mathrm{FT}_{4}$ Assay; its reference interval was 8.7-19.6 pmol/l, and was defined as described above. The interassay coefficients of variation were $11.1,11.3$ and $12.2 \%$ at concentrations of $6.1,19.3$ and $27.7 \mathrm{pmol} / \mathrm{l}$ respectively.

A free tri-iodothyronine ( $\mathrm{fT}_{3}$ ) assay for diagnosing a possible $\mathrm{T}_{3}$-toxicosis was performed in case of a decreased TSH but normal $\mathrm{fT}_{4}$ (reference interval: 4.0-8.0 pmol/l; Amerlex MAB, Amersham, Amersham, Bucks, UK).

Thyroid dysfunction (TD) was defined as an abnormal TSH (i.e. outside the reference interval), either in combination with an abnormal $\mathrm{fT}_{4}$ or an abnormal $\mathrm{fT}_{3}$ ('overt' $\mathrm{TD}$ ), or without an abnormal $\mathrm{fT}_{4}$ or $\mathrm{fT}_{3}$ ('subclinical' TD).

The study group was divided into three subgroups according to the observed thyroid function: I, no TD during the entire study period; II, TD at 12 and/or 32 weeks gestation (overt or subclinical); III, TD at one or more time points in the post partum period (overt (PPTD) or subclinical; women who had TD both in gestation and post partum were included in this group).

TPOAb were measured by using the Immunometric Enzyme Combikit (Orgentec GMBH, Mainz, Germany); a concentration of $>50 \mathrm{U} / \mathrm{ml}$ was defined as 'positive' $(\mathrm{TPOAb}+)$. The interassay coefficients of variation were 18 and $8.5 \%$ at concentrations of 18 and $1000 \mathrm{U} / \mathrm{ml}$ respectively.

For detection of thyrotropin receptor antibodies (TSH$\mathrm{RAb}$ ) in women with TD the TRAK-Assay (B.R.A.H.M.S. Diagnostica GMBH, Berlin, Germany) was used; a concentration of $\geq 15 \mathrm{U} / \mathrm{l}$ was considered positive.

Analysis Statistical analysis was performed by using the Statistical Package of Social Science (SPSS). 
Table 1 Thyroid dysfunction (TD; subclinical: TSH abnormal; overt: $\mathrm{TSH}$ and $\mathrm{fT}_{4}$ abnormal) in gestation or post partum in relation to the presence of TPOAb in gestation and/or in post partum.

\begin{tabular}{lccc}
\hline & $\begin{array}{c}\text { No TPOAb } \\
(\leq 50 \mathrm{U} / \mathrm{ml})\end{array}$ & $\begin{array}{c}\text { TPOAb } \\
(>50 \mathrm{U} / \mathrm{ml})\end{array}$ & Total $(\%)$ \\
\hline No TD & 212 & 20 & $232(80 \%)$ \\
$\begin{array}{l}\text { TD in gestation } \\
\quad \text { Subclinical }\end{array}$ & 14 & 1 & $15(5 \%)$ \\
$\quad$ Overt & 6 & 2 & $8(3 \%)$ \\
TD in post partum & & & \\
$\quad$ Subclinical & 13 & 8 & $21(7 \%)$ \\
$\quad$ Overt & 5 & 10 & $15(5 \%)$ \\
Total (\%) & $250(86 \%)$ & $41(14 \%)$ & $291(100 \%)$ \\
\hline
\end{tabular}

Differences between subgroups were analyzed by the Chi-square test or Student's t-test. RR with the corresponding 95\% confidence interval $(95 \% \mathrm{CI})$ for developing PPTD were calculated. Multivariate logistic regression analysis was performed to determine independent risk factors for PPTD; introduced into the model were all factors with $P \leq 0.1$ in the univariate analysis. Sensitivity, specificity and predictive values of TPOAb for detecting women at risk for PPTD at different time points and at different concentrations were calculated. Sensitivity and specificity were also presented in receiver operating characteristic (ROC) curves (with the corresponding area under the curve (AUC)).

Follow up All women who had experienced PPTD were visited again 2.5-3 years after delivery to perform thyroid function and TPOAb tests.

\section{Results}

From the 310 women participating in the study 19 (6.1\%) were excluded from analysis. The reasons for exclusion were: 7 women refused further participation after experiencing spontaneous miscarriage or stillbirth $(1 \mathrm{TPOAb}+), 1$ suffered from puerperal psychosis, 9 (1 $\mathrm{TPOAb}+$ ) were pregnant within 6 months after delivery and 2 moved out of the region. The study population was thus comprised of 291 women.

The different types of TD in relation to TPOAb are shown in Table 1: 232 women (80\%) did not develop TD, 23 women ( $8 \%$ ) had TD in gestation and 36 women (12\%) had TD post partum.

In Fig. 1 the results of the serial $\mathrm{fT}_{4}$ and $\mathrm{TSH}$ testing are presented. The mean $\mathrm{fT}_{4}$ and $\mathrm{TSH}$ levels were comparable to the values for non-pregnant women of the same age, with the exception of $\mathrm{fT}_{4}$ levels at 32 weeks gestation and TSH levels at 20 weeks post partum. Mean $\mathrm{fT}_{4}$ levels declined $22 \%$ during pregnancy, and returned to the original level within 12 weeks after delivery. Mean TSH

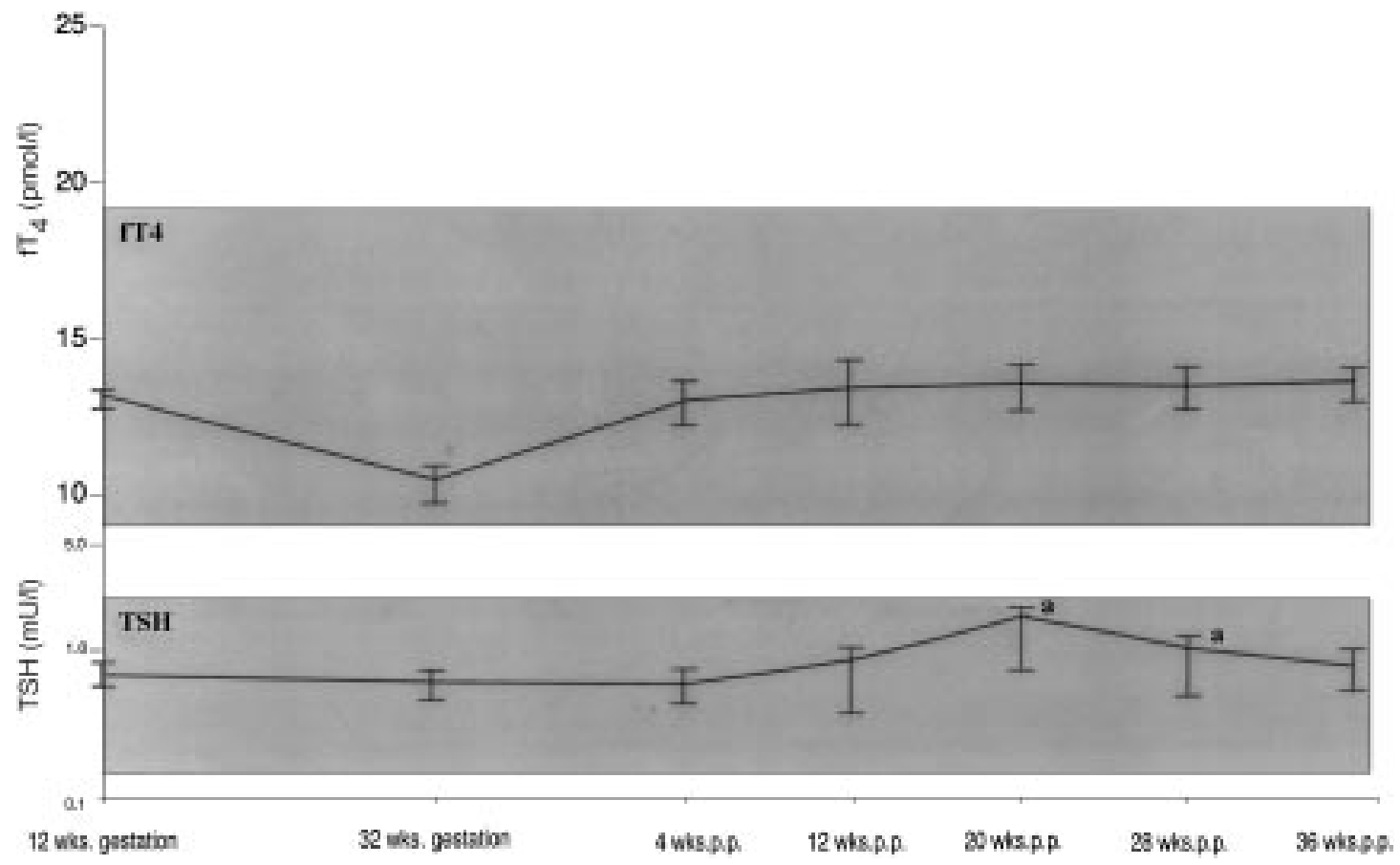

Figure 1 Results of serial $\mathrm{fT}_{4}$ and TSH testing in 291 women during gestation and post partum (p.p.). Values are means \pm S.E.M. Reference limits are $0.15-2.0 \mathrm{mU} / \mathrm{l}$ for TSH and $8.7-19.6 \mathrm{pmol} / \mathrm{l}$ for $\mathrm{fT}_{4}$ (shaded areas). ${ }^{\mathrm{a}} P<0.05$ versus 12 weeks gestation. 
concentrations remained stable during gestation, increased gradually to a peak at 20 weeks post partum, and then returned to the original level.

A total of 41 women $(14.1 \%$; see Table 1$)$ had TPOAb at one or more time points. Three patterns of TPOAb could be identified: 5 women had TPOAb only in gestation, 9 women had TPOAb (with increasing concentrations) only post partum and 27 women had TPOAb both in gestation and post partum. Figure 2 shows the point-prevalences of TPOAb at three different concentrations. The proportion of women with TPOAb $>50 \mathrm{U} / \mathrm{ml}$ at 12 weeks gestation was similar to that at $12,20,28$ and 36 weeks post partum $(10.0 \%$ versus $9.1 \%, 9.3 \%, 10.4 \%$ and $9.4 \%$ respectively). Both the point prevalence and concentration of TPOAb decreased during gestation. The proportion of women with concentrations of TPOAb $\geq 100$ or $\geq 300 \mathrm{U} / \mathrm{ml}$ increased considerably in the post partum period.

\section{Thyroid function in gestation}

TD in gestation was present in 23 women $(8 \% ; 3$ TPOAb+; see Table 1). Thirteen women had a decreased TSH concentration at 12 weeks, of whom 6 also had an increased $\mathrm{fT}_{4}$ concentration; the incidence of gestational thyrotoxicosis can thus be calculated as $2.1 \%(6 / 291)$. $\mathrm{TPOAb}$ were present in one woman with gestational thyrotoxicosis (and decreased TSH at 12 weeks post partum). Gestational thyrotoxicosis was not related to the development of PPTD. Ten women had increased TSH levels; of these 2 had marginally decreased $\mathrm{fT}_{4}$ levels.

The mean $\mathrm{fT}_{4}$ concentration during pregnancy was comparable for TPOAb- and TPOAb+ women; the mean TSH concentration, however, was significantly higher in TPOAb + women (at 12 weeks: 0.79 (s.E.M. $=$ 0.002 ) versus 1.10 (s.E.M. $=0.03) \mathrm{mU} / \mathrm{l}, P<0.001$; at 32 weeks: 0.76 (s.E.M. $=0.002$ ) versus 0.84 (s.E.M. $=$ $0.01) \mathrm{mU} / \mathrm{l}, \mathrm{P}<0.001)$.

\section{Thyroid function in the post partum period}

PPTD was present in 15 women (5.2\%; Table 1$)$. When subclinical TD was also taken into account, 36 women $(12.4 \%)$ had TD post partum (12 women (6 TPOAb+) had subclinical hypothyroidism and 9 subclinical hyperthyroidism $(2 \mathrm{TPOAb}+))$; from these 36 women $7(19.4 \%)$ also had TD at 12 weeks gestation (6 subclinical hypothyroidism and 1 subclinical hyperthyroidism). From the 15 women with PPTD, 9 had thyrotoxicosis without subsequent hypothyroidism (4 $\mathrm{TPOAb}+$ ), 5 hypothyroidism alone (all TPOAb+) and 1 thyrotoxicosis followed by hypothyroidism $(\mathrm{TPOAb}+)$. The $10 \mathrm{TPOAb}+$ women with PPTD all had decreasing $\mathrm{TPOAb}$ concentrations in gestation and increasing concentrations post partum. All women with PPTD were negative for TSH-RAb. TPOAb+ women with

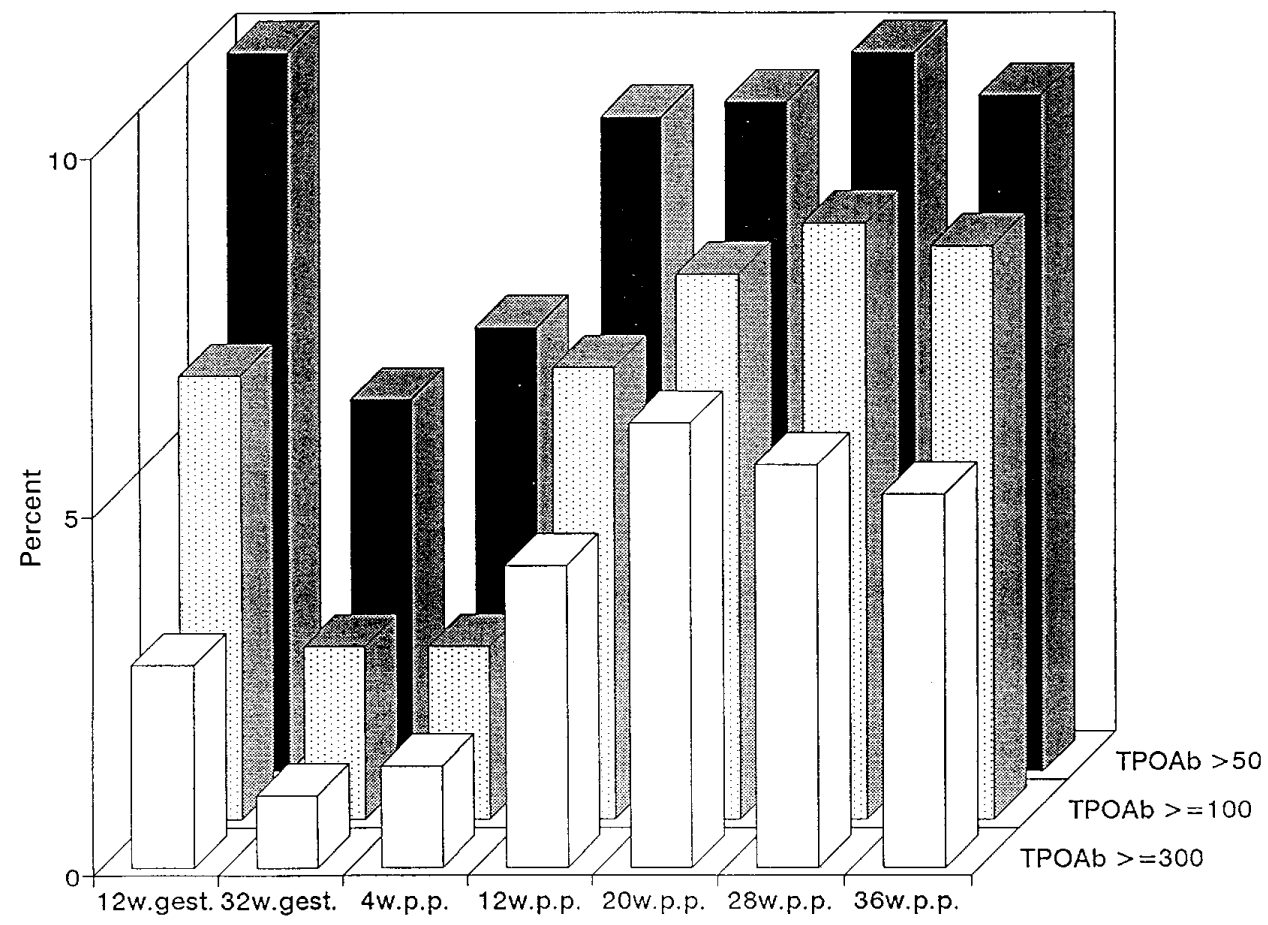

Figure 2 Point prevalences (\%) of TPOAb for three different concentrations at seven different time points (w.gest., weeks of gestation; w.p.p., weeks post partum). Point prevalence was calculated as the number of TPOAb positive women/total number of women $\times 100 \%$ for each time point separately. Total number of women $=291$. 
Table 2 The results of 2.5-3 years follow-up in 14 women who had experienced post partum thyroid dysfunction (TPOAb+: positive for TPOAb when PPTD occurred; TPOAb-: negative for TPOAb). Values are the mean (range) for $\mathrm{fT}_{4}$ and $\mathrm{TSH}$; other values, number of women.

\begin{tabular}{|c|c|c|}
\hline & $\begin{array}{l}\text { TPOAb+ } \\
(n=9)\end{array}$ & $\begin{array}{l}\text { TPOAb- } \\
(n=5)\end{array}$ \\
\hline $\begin{array}{l}\text { Thyroid function tests: } \\
\mathrm{fT}_{4}(\mathrm{pmol} / \mathrm{l}) \\
\mathrm{TSH}(\mathrm{mU} / \mathrm{l}) \\
\text { TPOAb+ }(>50 \mathrm{U} / \mathrm{ml})^{*}\end{array}$ & $\begin{array}{l}17(14-21)^{\text {** }} \\
3.2(0.9-5.4)^{\star *} \\
9\end{array}$ & $\begin{array}{l}19(14-22) \\
1.0(0.2-1.8) \\
0\end{array}$ \\
\hline $\begin{array}{l}\text { Thyroid (dys-)function } \\
\text { Hypothyroidism } \\
\text { Subclinical hypothyroidism } \\
\text { Normal } \\
\text { Hyperthyroidism }\end{array}$ & $\begin{array}{l}2 \\
4 \\
3 \\
0\end{array}$ & $\begin{array}{l}0 \\
0 \\
5 \\
0\end{array}$ \\
\hline
\end{tabular}

*TPOAB+; TPOAb present at follow up; ** 2 women on L-thyroxine treatment were excluded.

PPTD had more periods with signs and symptoms of hyper- and/or hypothyroidism than TPOAb- women with PPTD (13\% versus 4\%); two of these women were treated with L-thyroxine for several months because of symptoms of hypothyroidism. All women but one had normal TSH and $\mathrm{fT}_{4}$ concentrations at 36 weeks post partum. Fourteen of the fifteen women who had experienced PPTD were visited 2.5 to 3 years after delivery. The results of the thyroid function tests are summarized in Table 2. From the 9 TPOAb+ women, 2 had permanent hypothyroidism and were treated with L-thyroxine, 4 had increased TSH levels and 3 had normal thyroid function. The $5 \mathrm{TPOAb}-$ women, however, all had normal thyroid function.

\section{Prediction of PPTD}

None of the following parameters were related to the development of PPTD: age, educational level, alcoholuse during pregnancy, a family history of thyroid disease, parity, the number of pregnancy-related complications, or gender, congenital anomalies and birthweight of the child. Univariate analysis revealed that PPTD was significantly related to four parameters: (1) the presence of TPOAb: RR at 12 and 32 weeks gestation and 4 weeks post partum were 18.1 (95\%CI 6.6-49.3, $P<0.0001$ ), 27.6 (95\%CI 11.3-67.4, $P<$ $0.00001)$ and $22.8(95 \%$ CI 9.1-56.9, $P<0.00001)$ respectively; (2) TSH concentration $>2.0 \mathrm{mU} / \mathrm{l}$ at 12 weeks gestation: $\mathrm{RR}=5.4(95 \% \mathrm{CI} 1.7-16.7, P=0.02)$; (3) previous autoimmune disease (mainly AITD): $\mathrm{RR}=4.3$ (95\%CI 1.1-16.7, $P=0.03)$; (4) smoking habits ('ever smoked' (women who stopped smoking or were still smoking in gestation) versus 'never smoked'): $\mathrm{RR}=3.6(95 \% \mathrm{CI} 1.2-11.0, \mathrm{P}=0.016)$. Women with PPTD had smoked more cigarettes per day (46\% smoked $<10$ cigarettes/day, 27\% 10-20 cigarettes/day and $27 \%>20$ cigarettes/day versus $55 \%, 26 \%$ and $18 \%$
Table 3 Results of the multivariate regression analysis to determine independent factors related with post partum thyroid dysfunction expressed as RR $(95 \% \mathrm{Cl})$. Introduced into the model were factors with $P \leq 0.1$ in the univariate analysis.

\begin{tabular}{lc}
\hline & RR $(95 \% \mathrm{CI})$ \\
\hline Smoking habits (ever vs never) & $3.1(1.2-7.5)^{*}$ \\
Previous autoimmune disease & $2.0(0.1-37.7)$ \\
Family history of thyroid disease & $1.4(0.3-6.7)$ \\
Parity $\geq 1$ & $2.3(0.4-13.6)$ \\
Pregnancy complications & \\
$\quad$ Diabetes & $5.9(0.5-66.1)$ \\
$\quad$ Hypertension/toxicosis & $3.7(0.4-37.8)$ \\
Bottle feeding & $11.1(1.4-75.1)^{*}$ \\
TPOAb $>50 \mathrm{U} / \mathrm{ml}$ at 12 weeks gestation & $27.2(6.4-115.6)^{\star *}$ \\
\hline${ }^{*} P=0.04,{ }^{* *} P<0.0001$. &
\end{tabular}

respectively in women without PPTD; differences not significant) and for a longer period (mean: 12.9 $($ S.D. $=4.1)$ years versus $9.8 \quad($ S.D. $=3.8)$ years; $t$-test $P<0.05)$ than women without PPTD. The presence or absence of TPOAb was not related to smoking habits. Women with PPTD more frequently experienced diabetes or hypertension in pregnancy, and breast fed less, but these differences were not statistically significant $(0.05<P<0.1)$.

In order to determine independent factors related to PPTD a multivariate analysis was performed and three were found: TPOAb $(\mathrm{RR}=27.2)$, bottle feeding $(\mathrm{RR}=$ 11.1) and smoking habits ('ever smoked': $\mathrm{RR}=3.1$ ) (Table 3).

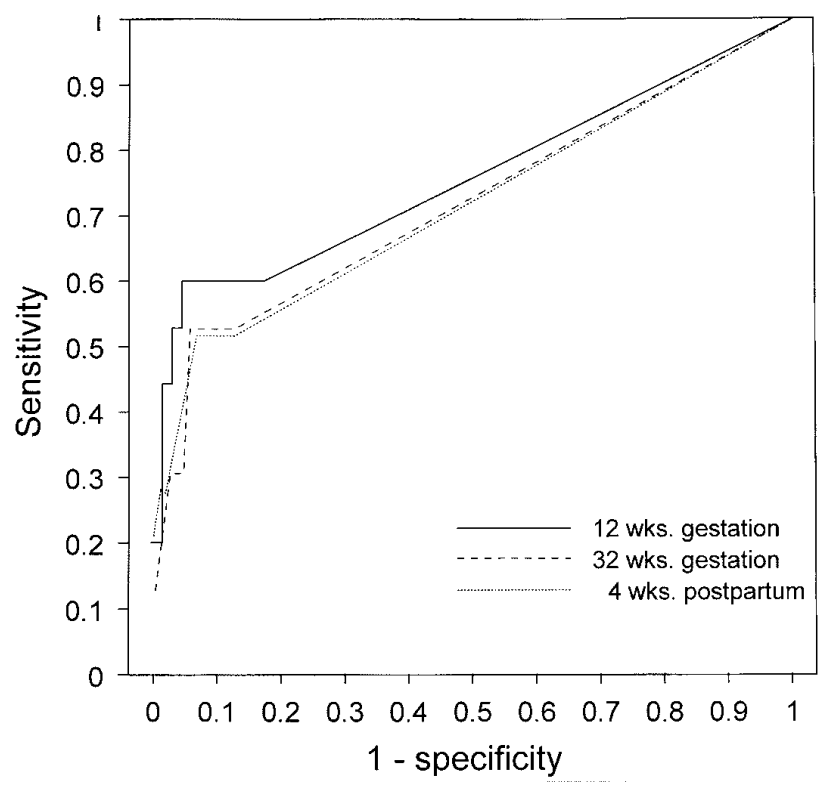

Figure 3 Receiver operating characteristic (ROC) curves for serum concentrations of TPOAb $>50 \mathrm{U} / \mathrm{ml}$ measured at 12 and 32 weeks gestation and 4 weeks post partum $(n=291)$. 
Table 4 Positive and negative predictive value, sensitivity and specificity of TPO-antibody testing at different time points and concentrations separately and in combination with independent anamnestic risk factors for developing post partum thyroid dysfunction.

\begin{tabular}{|c|c|c|c|c|}
\hline TPOAb & $\begin{array}{c}\text { Negative predictive } \\
\text { value }\end{array}$ & $\begin{array}{c}\text { Positive predictive } \\
\text { value }\end{array}$ & Sensitivity & Specificity \\
\hline $\begin{array}{l}\text { TPO }>5012 \text { weeks gestation } \\
\text { TPO } \geq 10012 \text { weeks gestation } \\
\text { TPO } \geq 30012 \text { weeks gestation }\end{array}$ & $\begin{array}{l}0.98 \\
0.98 \\
0.97\end{array}$ & $\begin{array}{l}0.31 \\
0.50 \\
0.75\end{array}$ & $\begin{array}{l}0.67 \\
0.67 \\
0.40\end{array}$ & $\begin{array}{l}0.93 \\
0.97 \\
0.99\end{array}$ \\
\hline $\begin{array}{l}\text { TPO }>5032 \text { weeks gestation } \\
\text { TPO } \geq 10032 \text { weeks gestation } \\
\text { TPO } \geq 30032 \text { weeks gestation }\end{array}$ & $\begin{array}{l}0.97 \\
0.99 \\
0.95\end{array}$ & $\begin{array}{l}0.64 \\
0.71 \\
0.67\end{array}$ & $\begin{array}{l}0.64 \\
0.33 \\
0.13\end{array}$ & $\begin{array}{l}0.97 \\
0.99 \\
0.996\end{array}$ \\
\hline $\begin{array}{l}\text { TPO }>504 \text { weeks post partum } \\
\text { TPO } \geq 1004 \text { weeks post partum } \\
\text { TPO } \geq 3004 \text { weeks post partum }\end{array}$ & $\begin{array}{l}0.97 \\
0.96 \\
0.96\end{array}$ & $\begin{array}{l}0.44 \\
0.63 \\
0.75\end{array}$ & $\begin{array}{l}0.53 \\
0.33 \\
0.20\end{array}$ & $\begin{array}{l}0.96 \\
0.99 \\
0.996\end{array}$ \\
\hline $\begin{array}{l}\text { TPO >50 } 12 \text { wks gest. + smoking } \\
\text { TPO >50 } 4 \text { wks p.p. + smoking } \\
\text { TPO >50 } 12 \text { wks gest. + bottle feeding } \\
\text { TPO >50 } 4 \text { wks p.p. + bottle feeding }\end{array}$ & $\begin{array}{l}0.97 \\
0.97 \\
0.97 \\
0.97\end{array}$ & $\begin{array}{l}0.50 \\
0.67 \\
0.38 \\
0.47\end{array}$ & $\begin{array}{l}0.47 \\
0.40 \\
0.53 \\
0.47\end{array}$ & $\begin{array}{l}0.97 \\
0.99 \\
0.95 \\
0.97\end{array}$ \\
\hline
\end{tabular}

wks gest., weeks of gestation; wks p.p., weeks post partum.

In Fig. 3 the ROC curves for TPOAb testing at 12 and 32 weeks gestation and 4 weeks post partum are shown. These ROC curves and their AUCs were comparable. The corresponding values of sensitivity and specificity are presented in Table 4 . The sensitivity of TPOAb testing alone was highest at 12 weeks gestation: all TPOAb+ women who developed PPTD had TPOAb $>50$ or $\geq 100 \mathrm{U} / \mathrm{ml}$ at that time point. The positive predictive value of TPOAb at each time point increased with increasing concentrations (Table 4 ). The positive predictive value of TPOAb $>50 \mathrm{U} / \mathrm{ml}$ was highest at 32 weeks gestation: the presence of TPOAb at that time point indicated a great probability of developing PPTD. The positive predictive values of higher TPOAb concentrations were similar for the different time points (Table 4). Combination of the results of TPOAb testing with the other independent risk factors (smoking habits and bottle feeding) increased the positive predictive value slightly (Table 4 ).

\section{Discussion}

Our study population consisted of a sample of pregnant women residing in the southeastern part of The Netherlands. Sampling bias is unlikely as at least $90 \%$ of the women in the region are attended by the local midwives or the obstetrical department of the St Joseph Hospital, Veldhoven; moreover mean age and parity of the non-participating women were comparable to those of the participants.

Low TSH concentrations in the first trimester and gestational thyrotoxicosis are thought to be the result of a TSH-like effect of human chorionic gonadotropin (hCG) (11, 16-19). In this study decreased TSH levels in early pregnancy were present in $4.5 \%$ and gestational thyrotoxicosis in $2.1 \%$ of subjects. Glinoer et al. showed that in a region with restricted iodine intake undetectable
TSH levels were found in $13 \%$ of subjects and increased $\mathrm{fT}_{4}$ levels were found in $3 \%$ of subjects in the first trimester of pregnancy, and in a later study a prevalence for gestational thyrotoxicosis was found in $2.4 \%$ of subjects $(11,18)$. The decline of $\mathrm{fT}_{4}$ during pregnancy and return to baseline levels post partum (Fig. 1) has been described previously $(11,17,18,20)$. The mechanism that causes the decline of $\mathrm{fT}_{4}$ without a concomitant rise of TSH during pregnancy is still unknown $(11,17)$.

The prevalence and course of TPOAb concentration and the incidence of PPTD were in accordance with the results of other studies $(3,5,6,21,22)$.

Although $\mathrm{fT}_{4}$ concentrations in gestation in $\mathrm{TPOAb}+$ women were similar to those of TPOAb- women TSH concentrations were slightly but significantly higher in TPOAb + women. This is in accordance with the findings of Glinoer et al. who reported that TPOAb+ women are at risk for developing (subclinical) hypothyroidism during pregnancy because of the reduced functional capacity of the thyroid $(10,11)$.

Our study confirmed that TPOAb are the most prominent risk factor for PPTD $(4-6,8,9,12,21-$ 24). RR were 18,28 and 23 at 12 and 32 weeks gestation and 4 weeks post partum respectively; this is in accordance with a previous study from the same region (5); Gerstein, however, calculated a $R R=87$, probably due to the use of less sensitive MsAb tests in the 1970 s and 1980s (when TPO $\geq 100 \mathrm{U} / \mathrm{ml}$ is taken as the cut off point the RR increases to 18,45 and 31 at 12 and 32 weeks gestation and 4 weeks post partum respectively) (3).

Women who had ever smoked had an increased risk for developing PPTD (RR=3.1). Fung et al. also found that smoking was related to PPTD (24); others, however, did not find smoking related to PPTD or autoimmune hypothyroidism $(21,25)$. Arguments in favor of this 
relation are: the possible dose-response effect that we found, the noxious effect of diverse components of tobacco on the thyroid and the effect of smoking on the immune system $(26,27)$.

This is the first study describing breast feeding as an independent protecting factor: women not breast feeding had a $\mathrm{RR}=11.1$ for developing PPTD. In the studies from Jansson et al. and Fung et al. PPTD was not related to lactation $(21,24)$. The protective effect cannot be explained by the prolonged high prolactin levels during breast feeding: hyperprolactinemia has both immunosuppressive and immunostimulative effects and is associated with the presence of thyroid antibodies (28). Possible explanations are: some other still unknown immunomodulating factors associated with pregnancy which remain operative during lactation, or selection (women prone to PPTD would not start breast feeding).

Anamnestic factors independently related to PPTD (smoking habits, bottle feeding) had limited additional value for predicting PPTD. The positive predictive value increased slightly, but the sensitivity decreased, which resulted in the identification of a small group of women at risk for developing PPTD with more accuracy, but a large number of cases would be missed (Table 4).

One-third of the women developing PPTD had no TPOAb. All these women experienced only a phase of hyperthyroidism post partum with slight or no complaints. PPTD characterized by hyperthyroidism alone in the absence of both MsAb and TSH-RAb has previously been described $(24,29)$. The authors supposed that this form of PPTD was not the result of an exacerbation of AITD but had a different etiology, although in some cases thyroiditis was found in fine needle aspiration biopsy (29). The proportion of women with this form of (non-autoimmune) PPTD can be estimated as $10-30 \%$ of all PPTD cases $(3,5,24)$. In contrast, the $10 \mathrm{TPOAb}+$ women who developed PPTD had more complaints (such as lack of energy and depression) and 2 women needed transient thyroxine replacement therapy. This form of PPTD is the 'classical' form presenting with hyperthyroidism followed by hypothyroidism and is caused by exacerbation of a pre-existing AITD. These women with high TSH levels in combination with TPOAb appear to be really at risk for developing permanent hypothyroidism (Table 2) (30-32).

We suppose that especially PPTD in TPOAb+ women (the 'classical form' with the 'classical pattern' of TPOAb decreasing in gestation and increasing post partum) is of clinical interest. Identification of these women with great accuracy is important when an intervention (e.g. treatment with thyroxine) to prevent PPTD is considered, but also because of the considerable risk of developing permanent hypothyroidism $(30,31)$. Therefore, we calculated the positive predictive values and sensitivity and specificity for TPOAb concentrations $\geq 100$ and $\geq 300 \mathrm{U} / \mathrm{ml}$ respectively for TPOAb + women at different time points. The positive predictive values remained unchanged and sensitivity increased: the chance of developing PPTD did not alter but TPOAb+ women at risk were detected more accurately.

The most appropriate moment for screening for $T P O A b$ is in the first trimester of pregnancy if a test with the highest sensitivity is required; on the other hand, if a test with high predictive value is wanted testing should be performed in the third trimester or 1 month post partum: when a woman is positive for TPOAb at that time point she has a great chance of developing the autoimmune form of PPTD (see Table 4).

In conclusion, the results of this study indicate that combination of TPOAb testing with independent determinants such as smoking habits and bottle feeding has limited additional value for predicting PPTD. There appeared to be two forms of PPTD: an autoimmune form which occurred in two-thirds of cases and a nonautoimmune form. Intervention with thyroxine to prevent symptoms of PPTD, and long-term follow up for detecting permanent hypothyroidism should be considered only in women with the autoimmune form of PPTD. Further research to evaluate the effects of treatment with thyroxine, and to study the etiology and long-term effects of the non-autoimmune form is needed.

\section{Acknowledgements}

This study was supported by grants from the Dutch Praeventiefonds (no: 002822380) and the Dr de Grood Foundation. We thank Marco van de Paal for preparing the figures, and Kodak Clinical Diagnostics Ltd, Amersham, UK, for the TSH and $\mathrm{fT}_{4}$ kits and Diagnostic Products Co. Nederland bv. for the TPOAb kits.

\section{References}

1 Amino N, Miyai K, Onishi T, Hashimoto T, Arai K, Ishibashi K et al. Transient hypothyroidism after delivery in autoimmune thyroiditis. Journal of Clinical Endocrinology and Metabolism 197642 296301.

2 Ginsberg J \& Walfish PG. Post-partum transient thyrotoxicosis with painless thyroiditis. Lancet 1977 i 1125-1128.

3 Gerstein HC. How common is postpartum thyroiditis? Archives of Internal Medicine 1990150 1397-1400.

4 Lazarus JH \& Othman S. Thyroid disease in relation to pregnancy. Clinical Endocrinology 199134 91-98.

5 Pop VJM, Rooy HAM de, Vader HL, Heide D van der, Son MM van \& Komproe IH. Microsomal antibodies during gestation in relation to postpartum thyroid dysfunction and depression. Acta Endocrinologica 1993129 26-30.

6 Amino N, Tada H \& Hidaka Y. Autoimmune thyroid disease and pregnancy. Journal of Endocrinological Investigation 199619 59-70.

7 Harris B, Othman S, Davies JA, Weppner GJ, Richards CJ, Newcombe RG et al. Association between postpartum thyroid dysfunction and thyroid antibodies and depression. British Medical Journal 1992305 152-156.

8 Lazarus JH, Hall R, Othman S, Parkes AB, Richards CJ, McCulloch $\mathrm{B}$ et al. The clinical spectrum of postpartum thyroid disease. Quarterly Journal of Medicine 199689 429-435. 
9 Stagnaro-Green A, Roman SH, Cobin RH, El-Hazary E, Wallenstein S \& Davies TF. A prospective study of lymphocyte-initiated immunosuppression in normal pregnancy: evidence of a T-cell etiology for postpartum thyroid dysfunction. Journal of Clinical Endocrinology and Metabolism 199274 645-653.

10 Glinoer D, Riahi M, Gruen J-P \& Kinthaert J. Risk of subclinical hypothyroidism in pregnant women with asymptomatic autoimmune thyroid disorders. Journal of Clinical Endocrinology and Metabolism 199479 197-204.

11 Glinoer D. The regulation of thyroid function in pregnancy: pathways of endocrine adaptation from physiology to pathology. Endocrine Reviews 199718 404-433.

12 Weetman AP. Prediction of post-partum thyroiditis. Clinical Endocrinology 199441 7-8.

13 Ball S. Antenatal screening of thyroid antibodies. Lancet 1996 348 906-907.

14 Rees-Wortelboer MM van, Schroeder-van der Elst JP, Lycklama A \& Heide D van der. Jodium en krop in Nederland. Nederlands Tijdschrift voor Geneeskunde 1987131 1821-1824.

15 Solberg HE \& Graessbeck R. Reference values. Advances in Clinical Chemistry 198927 1-79.

16 Pekonen F, Alfthan H, Stenman U-H \& Ylikorkala O. Human chorionic gonadotropin (hCG) and thyroid function in early human pregnancy: circadian variation and evidence for intrinsic thyrotropic activity of hCG. Journal of Clinical Endocrinology and Metabolism $198866853-856$.

17 Wiersinga WM, Vet T, Berghout A \& Endert E. Serum free thyroxine during pregnancy: a meta-analysis. In The Thyroid and Pregnancy, pp 79-94. Eds C Beckers \& D Reinwein. New York: Schattauer, 1991.

18 Glinoer D, Nayer P de, Bourdoux P, Lemone M, Robyn C, Steirteghem A van et al. Regulation of maternal thyroid during pregnancy. Journal of Clinical Endocrinology and Metabolism 1990 $71276-287$.

19 Kraiem Z, Sadeh O, Blithe DL \& Nisula BC. Human chorionic gonadotropin stimulates thyroid hormone secretion, iodide uptake, organification, and adenosine $3^{\prime} 5^{\prime}$-monophosphate formation in cultured human thyrocytes. Journal of Clinical Endocrinology and Metabolism 1994 79 595-599.

20 Glinoer D, Lemone M, Bourdoux P, Nayer P de, Delange F, Kinthaert J et al. Partial reversibility during late postpartum of thyroid abnormalities associated with pregnancy. Journal of Clinical Endocrinology and Metabolism 199274 453-457.

21 Jansson R, Bernarder S, Karlsson A, Levin K \& Nilsson G. Autoimmune thyroid dysfunction in the postpartum period. Journal of Clinical Endocrinology and Metabolism 198458 681-687.

22 Lervang H-H, Pryds O \& Ostergaard Kristensen HP. Thyroid dysfunction after delivery. Incidence and clinical course. Acta Medica Scandinavia 1987222 369-374.

23 Amino N, Mori H, Iwatani Y, Tanizawa O, Kawashima M, Tsuge I et al. High prevalence of transient post-partum thyrotoxicosis and hypothyroidism. New England Journal of Medicine 1982306 849852 .

24 Fung HYM, Kologlu M, Collison K, John R, Richards CJ, Hall R et al. Postpartum thyroid dysfunction in Mid Glamorgan. British Medical Journal 1988296 241-244.

25 Prummel MF \& Wiersinga WM. Smoking and risk of Graves' disease. Journal of the American Medical Association 1993269 479-482.

26 Utiger RD. Cigarette smoking and the thyroid. New England Journal of Medicine 1995333 1001-1002.

27 George J, Levy Y \& Shoenfeld Y. Smoking and immunity: an additional player in the mosaic of autoimmunity. Scandinavian Journal of Immunology 199745 1-6.

28 Reber PM. Prolactin and immunomodulation. American Journal of Medicine $199395637-644$.

29 Dahlberg PA \& Jansson R. Different aetiologies in post-partum thyroiditis? Acta Endocrinologica 1983104 195-200.

30 Tachi J, Amino N, Tamaki H, Aozasa M, Iwatani Y \& Miyai K. Long term follow-up and HLA association in patients with postpartum hypothyroidism. Journal of Clinical Endocrinology and Metabolism $198866480-484$.

31 Othman S, Phillips DIW, Parkes AB, Richards CJ, Harris B, Fung H et al. A long-term follow-up of postpartum thyroiditis. Clinical Endocrinology 199032 559-564.

32 Vanderpump MPJ, Tunbridge WMG, French JM, Appleton D, Bates D, Clark F et al. The incidence of thyroid disorders in the community: a twenty-year follow-up of the Whickham Survey. Clinical Endocrinology 199543 55-68.

Received 19 December 1997

Accepted 23 February 1998 